\title{
Calculation of the number of partitions with constraints on the fragment size
}

\author{
P. Désesquelles \\ Institut de Physique Nucléaire, Bt. 100, 15 rue Georges Clémenceau, \\ F91406 Orsay Cedex, France
}

\begin{abstract}
This article introduces recursive relations allowing the calculation of the number of partitions with constraints on the minimum and/or on the maximum fragment size. PACS numbers: 02.10.De, 05.10.-a, 64.60.Ak
\end{abstract}

\section{INTRODUCTION}

A partition is an ensemble of positive integers with a given sum?. A partition can also be seen as a way to break a piece of discret matter into fragments. The number of partitions of a given integer is a quantity which is useful in various fields. In the so-called minimal information model [1] for example, all the partitions have the same probability $P(\mathbf{n})=1 / N(S)$. This result is obtained by application of the minimal information principle (or maximum entropy), information being defined as $\sum_{\mathbf{n}} P(\mathbf{n}) \ln P(\mathbf{n})$. When this equation is differentiated under the only constraint $\sum_{s} s n_{s}=S$, all probabilities are found to be equal 2.

\section{A. Physical Interest}

In many cases, it is interesting to distinguish one or more classes of fragments according to their size. In percolation, for instance, the sub-critical events are defined by the fact that they contain one particular fragment which is refered to as infinite or percolative in the sense that it connects the two extremes of the lattice 3. In the case of conductive bonds, it allows an electric current to circulate between two electrodes placed on opposite surfaces of the lattice. In the case of a coffee-machine, the percolative cluster defines a path which allows the vapor to traverse the grounds. In Nuclear Physics, other classes of fragments are distinguished. The intermediate mass fragments (IMF) are ions resulting from the violent fragmentation of a composite nucleus (produced by the collision of two

\footnotetext{
${ }^{1}$ For example, the integer 3 has 3 partitions : $\{3\},\{2,1\}$ and $\{1,1,1\}$.

${ }^{2}$ In the following, the total number of partitions of the integer $S$ will be noted $N(S)$, a partition will be noted $\mathbf{n}$ : $\left(n_{1}, \ldots, n_{S}\right), n_{s}$ being the number of integers $s$ in the partition.
}

atomic nuclei). When the nucleus is weakly excited, it "evaporates" some light particles so that, at the end of the process, we are left with light fragments and a heavy evaporation residue which contains almost all the charge of the initial nucleus. At very high excitation energies, the nucleus is completely "vaporized" into light fragments. In the energy range between these two extremes, the nucleus undergoes "multifragmentation" into a large number of fragments of all sizes. The multifragmentation process is thus characterized by the production of IMF. Some multifragmentation models consider the light fragments (with charge less than or equal to 2) as nuclear matter in the gazeous phase whereas the evaporation residue or the IMF form the liquid phase [4]. This terminology is also used in the field of phase transition models. Thus, in various fields, classes of fragments are defined by their size. Hence it is of interest to enumerate the number of partitions with constraints on the fragment size.

In Section I B some techniques used to calculate the number of partitions of an integer without conditions on the size of the fragments will be reviewed. It will then be shown how the partitions with constraints on the maximum size of the fragments (Section II A), on the minimum size of the fragments (Section II B) and on a size range (Section $\amalg \mathrm{IO}$ ) can be enumerated.

\section{B. The number of unconstrained partitions}

The total number of partitions of the integer $S$ is given approximatively by the Ramanujan-Hardy [5] formula which leading term is :

$$
N(S) \approx \frac{\exp \left(\pi \sqrt{\frac{2 S}{3}}\right)}{4 S \sqrt{3}} .
$$

As can be seen, the number of partitions increases very rapidly with $S$. The exact value of the number of partitions can be obtained using one of the following recursive formulae (the number of partitions of $S$ into $M$ fragments is noted $N(S, M)$ and $M$ is referred to as the multiplicity) :

$$
\begin{aligned}
N(S, M) & =N(S-1, M-1)+N(S-M, M) \\
& =\sum_{m=1}^{M} N(S-M, m) .
\end{aligned}
$$

From this relation [6], the equation giving the total number of partitions can be deduced : 


$$
N(S)=1+\sum_{M=2}^{S} \sum_{k=0}^{\operatorname{Int}(S / M)-1} N(S-k M-1, M-1) .
$$

The Euler [7] recursive relation leads to the same result :

$$
\begin{array}{r}
N(S)=\sum_{k=1}(-1)^{k+1}\left(N\left(S-\frac{3 k^{2}-k}{2}\right)\right. \\
\left.+N\left(S-\frac{3 k^{2}+k}{2}\right)\right) .
\end{array}
$$

\section{NUMBER OF PARTITIONS WITH CONSTRAINTS ON THE SIZE OF THE FRAGMENTS}

\section{A. Constraint on the maximum size}

The number of partitions of an integer $S$ into $M$ fragments with size less than or equal to $s_{\max }$ will be noted ${ }^{s_{\max }} N(S, M)$. It is obtained using a modified version of the recursive relation (2) :

$$
\begin{array}{r}
s_{\max } N(S, M)={ }^{s_{\max }} N(S-1, M-1) \\
+{ }^{s_{\max }} N(S-M, M) \\
-{ }^{s_{\max }} N\left(S-M-s_{\max }, M-1\right) \\
\text { if } \quad S \leq \frac{M\left(s_{\max }+1\right)}{2}, \\
s_{\max } N(S, M)={ }^{s_{\max }} N\left(M\left(s_{\max }+1\right)-S, M\right) .
\end{array}
$$

The boundary condition is :

$$
s_{\max } N(0,1)=1 .
$$

These relations lead to the calculation of $s_{\max } N$ for any value of $S$ and $M$. The three right hand terms in Eq. (6) are explained as follows. The partition ensemble can be shared into two sub-groups. The first one contains all the partitions including at least one size 1 fragment. One of these size 1 fragments can be removed from each partition in the sub-group. It follows that the number of partitions in the sub-group can be written as ${ }^{s_{\max }} N(S-1, M-1)$. The second group includes the partitions with no size 1 fragment. Hence one unit can be removed from each fragment without modifying the multiplicity. In the absence of any condition on the maximum size, the number of partitions in the second group would be $N(S-M, M)$. However, among the partitions into $M$ fragments of the integer $S-M$, some have one or more fragments with size $s_{\max }$. It is not possible to add one unit to these fragments, thus the corresponding partitions should not be counted. The number of these invalid partitions is obtained by removing one fragment with size $s_{\max }$. The other fragments can have any size less than or equal to $s_{\max }$ and their multiplicity is $M-1$. The number of invalid partitions is thus ${ }^{\max } N(S-$ $\left.M-s_{\max }, M-1\right)$.

The symmetry relation (7) can be demonstrated graphically using the so-called Ferrers diagram in which a size $s$ fragment is representated by a column of $s$ dots and a partition by its set of fragments sorted in a decreasing order. We complete the Ferrers diagrams with open dots as indicated in figure 1. The number of partitions of the integer $S$ into $M$ parts with sizes less than or equal to $s_{\max }$ is equal to the number of ways of arranging the dots in the thin line boxe. As the multiplicity is fixed, the bottom row is necessarily full. By a $180 \mathrm{deg}$ rotation the open dots play the same role as the black dots. The open dots partition will be refered to as complementary partition of the black dots (which should not be confused with the conjugate partition which is obtained by inverting $M$ and $s_{\max }$ ). Thus, to each multiplicity $M$ partition of the integer $S$ corresponds exactly one multiplicity $M$ partition of the $M\left(s_{\max }+1\right)-S$ open dots.
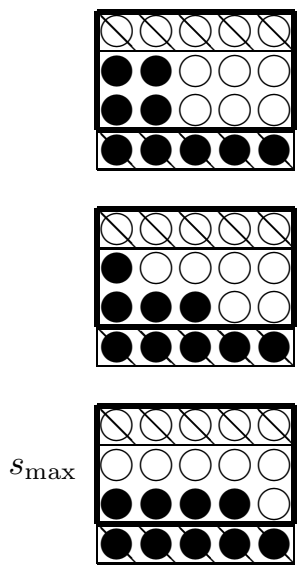

M

FIG. 1. Graphical sketchs of the three partitions $(\{3,3,1,1,1\},\{3,2,2,1,1\},\{2,2,2,2,1\})$ of the integer 9 into 5 fragments with size less than or equal to 3 (thin line boxes). The bold line boxes contain all the complementary partitions $(\{3,3,3,1,1\},\{3,3,2,2,1\},\{3,2,2,2,2\})$ of the 11 open dots with the same multiplicity and maximum size. The hatched dots do not participate in the enumeration of partitions (due to the multiplicity constraint).

For example, in the frame of the minimal information model, it can be interesting to know the number of partitions containing a given set $\mathbf{N}$ : $\left(N_{s_{\min }}, \ldots, N_{S}\right)$ of "large" fragments (i.e. fragments with size greater or equal to $s_{\min }$ ) supplemented by "small" fragments : 


$$
N(\mathbf{N})={ }^{s_{\min }-1} N\left(S-\sum_{s=s_{\min }}^{S} s N_{s}\right)
$$

An alternative method for enumerating the partitions with constraint on the maximum size consists in using the equivalent of equation (3) which takes the following form in this case :

$$
\left.s_{\max } N(S, M)=\sum_{m=1}^{M} s_{\max }-1 \text { N } N-M, m\right) .
$$

This equation can be applied recursively $s_{\max }-1$ times so that the maximum size in the right hand term is 1 . Using ${ }^{1} N(S, M)=1$ if $S=M$ and 0 otherwise, one obtains :

$$
s_{\max } N(S, M)=\sum_{m_{1}} \ldots \sum_{m_{k}=\operatorname{Int}\left(\frac{R_{k}-1}{s_{\max }-k}\right)+1}^{\operatorname{Min}\left(m_{k-1}, R_{k}\right)} \cdots \sum_{m_{s_{\max }-2}} 1
$$

FIG. 2. Ferrers diagrams illustrating the determination of the minima and maxima of the sums in equation (11). The goal is to determine the range of $m_{2}$ when $m_{0}=m_{1}=4$ and $S=11$ (thus $R_{2}=3$ ). The fragments are sorted in decreasing order, thus $m_{2}\left(s_{\max }-2\right) \geq R_{2}$, that is, the minimum value of $m_{2}$ is 2 . The number of fragments with size greater than $k$ is necessarily lower than the number of fragments with size greater than $k-1$, thus $m_{2} \leq m_{1}$. Furthermore, their are only $R_{2}$ units left, thus $m_{2} \leq R_{2}$. Finally, the maximum value for $m_{2}$ is the minimum of $m_{1}$ and $R_{2}$ (i.e. 3). More generally, the sum for $m_{k}$ runs from $\operatorname{Int}\left(\frac{R_{k}-1}{s_{\max }-k}\right)+1$ to $\operatorname{Min}\left(m_{k-1}, R_{k}\right)$.

Using the same line of thought on the conjugate partition, one obtains the following equation :

$$
s_{\max } N(S, M)=\sum_{s_{1}} \ldots \sum_{s_{k}=\operatorname{Int}\left(\frac{R_{k}-1}{M-k+1}\right)+1}^{\operatorname{Min}\left(s_{k-1}, R_{k}-M+1\right)} \cdots \sum_{s_{M-1}} 1,
$$

with $m_{0}=M$ and $R_{k}=S-\sum_{i=0}^{k-1} m_{i}$. In this equation, $m_{k}$ is the multiplicity of fragments with size strictly greater than $k$. The determination of the range for $m_{k}$ is illustrated in Fig. 22.

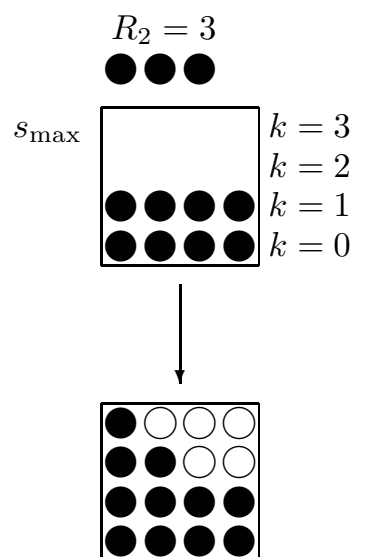

$m_{2}=2$

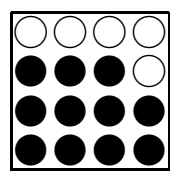

$m_{2}=3$ with $s_{0}=s_{\max }$ and $R_{k}=S-\sum_{i=1}^{k-1} s_{i}, s_{i}$ being the size of the $i^{\text {th }}$ largest fragment. The same equations hold for $N(S, M)$, fixing $s_{\max }=S$.

\section{B. Constraint on the minimum size}

The number of partitions of the integer $S$ into $M$ fragments with size greater or equal to $s_{\min }$ will be noted ${ }_{s_{\text {min }}} N(S, M)$. In each event, $M s_{\min }$ units are imposed (in Fig. 3 they correspond to the two lower rows). The number of partitions only depends on the $S-M s_{\text {min }}$ remaining units, for multiplicities ranging from 1 to $M$ :

$$
s_{\text {min }} N(S, M)=\sum_{m=1}^{M} N\left(S-M s_{\min }, m\right) .
$$

Following Eq. (3), this expression can be simplified to :

$$
s_{\min } N(S, M)=N\left(S-M\left(s_{\min }-1\right), M\right) .
$$

The same property can be directly deduced by considering the complementary partition (see open dots in Fig. 3). 
$s_{\text {min }}=2$

$M=3$
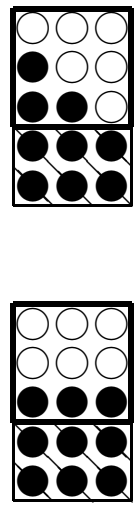

FIG. 3. Graphical sketchs of all the partitions ( $\{5,2,2\}$, $\{4,3,2\},\{3,3,3\})$ of the integer 9 into 3 fragments with size greater or equal to 2 . The two lower rows play no role in the counting of the partitions. The bold line box includes all the partitions of the integer 3 (i.e. $S-M s_{\min }$ ) with multiplicities less than or equal to 3 .

The total number of partitions is :

$$
s_{\min } N(S)=\sum_{M=1}^{S / s_{\min }} N\left(S-M\left(s_{\min }-1\right), M\right) .
$$

The boundary conditions are :

$$
\begin{array}{ll}
N(0, M \neq 1) & =0 \text { and } \\
N(0,1) & =1
\end{array}
$$

\section{Constraint on the minimum and maximum sizes}

When both the minimum and the maximum size of the fragments are fixed, the counting of the partitions is carried out in the same way as previously : $M s_{\min }$ units play no role. The number of partitions is the same as that of the integer $S-M s_{\min }$ into fragments with size less than or equal to $s_{\max }-s_{\min }$ (Fig. (4). Thus, the number of doubly-conditioned partitions is obtained as a sum over the number of singly-conditioned partitions.

$$
{ }_{s_{\min }}^{s_{\max }} N(S, M)=\sum_{m=1}^{M} s_{\max }-s_{\min } N\left(S-M s_{\min }, m\right)
$$

An alternative expression can be obtained by considering the complementary partitions (see Fig. (1) :

$$
{ }_{s_{\min }}^{s_{\max }} N(S, M)=\sum_{m=1}^{M} s_{\max }-s_{\min } N\left(M s_{\max }-S, m\right) .
$$

Applying Eq. (10) to (17) and (18) one obtains respectively :

$$
\begin{aligned}
s_{\max } N(S, M) & ={ }^{s_{\max }-s_{\min }+1} N\left(S-M\left(s_{\min }+1\right), M\right), \\
& ={ }^{s_{\max }-s_{\min }+1} N\left(M\left(s_{\max }+1\right)-S, M\right) .
\end{aligned}
$$

Finally :

$$
s_{\max } N(S)=\sum_{M=1}^{S / s_{\min }} s_{\max } s_{\min } N(S, M)
$$

$$
s_{\text {max }}=5
$$

FIG. 4. Graphical sketch of one partition $(\{5,4,3,2\})$ of the integer 14 into fragments with size included between 2 and 5. The two lower rows play no role in the partition counting. The bold line box contains all the partitions of the integer 6 (i.e. $S-M s_{\min }$ ) into fragments with size less than or equal to 3 and multiplicity less than or equal to 4 .

\section{CONCLUSION}

In this article, we have provided formulae for the calculation of the number of partitions with conditions on the maximum fragment size (Eq. (6.7)), with conditions on the minimum fragment size (Eq. (15) ) and with conditions on both the minimum and the maximum fragment size (Eq. (1920)). To demonstrate these formulae, the notion of complementary partitions was introduced. The constrained partition numbers are notably useful in the analysis of nuclear multifragmentation. Moretto and collaborators [8] have introduced an elegant combinatorial procedure to isolate rare events corresponding to the fragmentation of the atomic nucleus in a number of nearly equal size IMF (fragments with charge greater or equal to $Z_{\min }$ ) supplemented by light fragments (fragments with charge less than or equal to $Z_{\min }-1$ ). This procedure requires the evaluation of the number of partitions corresponding to a given sum $\left(Z_{\mathrm{imf}}\right)$ of the charges of a given number $(M)$ of IMF. This number of partitions is given as $Z_{\min } N\left(Z_{\mathrm{imf}}, M\right)^{Z_{\min }-1} N\left(Z_{\mathrm{tot}}-Z_{\mathrm{imf}}\right)$. The total number of partitions can be evaluated by the following convolution $N(S)=\sum_{s s_{\min }} N(s){ }^{s_{\min }-1} N(S-s)$. In a forthcomming article [9] we will show how the 
Moretto charge correlation can be calculated explicitly in the frame of the minimal information model. More generally these formulae are useful in domains where the fragment classes (infinite fragments, evaporation residues, light particles, intermediate mass fragments, liquid and gazeous phases...) are defined with respect to their sizes.

[1] R. Engelman, Phil. Mag. B50 (1984) 307.

R. Engelman, J. Phys. G5 (1991) 1019.

A.S. Botvina, A.D. Jackson and I.N. Mishustin, Phys. Rev. E62 (2000) R64.

[2] J. Aichelin and J. Huefner, Phys. Let. B136 (1984) 15. J. Aichelin et al., Phys. Rev. C30 (1984) 107.

[3] D. Stauffer, Introduction to Percolation Theory, Taylor and Francis, London (1985).

$\mathrm{X}$. Campi, Introduction à la théorie des modèles de formations d'amas, $107^{\text {th }}$ course of International School of Physics "Enrico Fermi" on the Chemical Physics of Atomic and Molecular Clusters. Varenne (1988)165.

[4] A.J. Cole, Statistical Models for Nuclear Decay, Fundamental and Applied Nuclear Physics Series, IOP (Bristol, Philadelphia) (2000).

[5] M. Abramowitz \& I. Stegun, Handbook of Mathematical functions, NBS, Applied Math Series-55 (U.S. GPO, Washington, DC) (1965).

[6] J.P. Bondorf et al., Nucl. Phys. A443 (1985) 321.

[7] J. Riordan, An Introduction to Combinatorial Analysis (Wiley, New York, 1967), p 117.

L.G. Sobotka and L.G. Moretto, Phys Rep. C31 (1984) 668.

[8] L.G. Moretto et al., Phys. Rev. Let. 77 (1996) 2634, L.G. Moretto et al., Phys. Rep. 287 (1997) 249.

[9] P. Désesquelles, submitted to Phys. Rev. C. 